\title{
Interaçōes entre Síndrome da Apnéia Obstrutiva do Sono e Resistência à Insulina
}

\begin{abstract}
RESUMO
Estudos anteriores mostraram que pacientes com Apnéia Obstrutiva do Sono (AOS) apresentam maior risco para doenças cardiovasculares. Entretanto, permanece controverso se essa associação depende da obesidade ou se ocorre devido a alterações fisiológicas decorrentes da desordem do sono, como ativação do sistema nervoso simpático, da inflamação e desordens do eixo corticotrófico e somatotrófico, que predispõem a danos vasculares. Além disso, muitos fatores de risco para doenças cardiovasculares (DCV) estão fortemente associados ao distúrbio respiratório, entre eles hipertensão, obesidade, resistência à insulina e diabetes tipo 2 (DM2). Neste artigo, vamos discutir a interação entre resistência à insulina e AOS e os possíveis mecanismos fisiopatológicos que contribuem para suas co-morbidades. (Arq Bras Endocrinol Metab 2007;51/7:1035-1040)
\end{abstract}

Descritores: Apnéia do sono; Resistência à insulina; Doenças cardiovasculares

\section{ABSTRACT}

\section{Interactions Between Obstructive Sleep Apnea Syndrome and Insulin Resistance.}

Previous studies have shown Obstructive Sleep Apnea (OSA) as a risk factor for development of cardiovascular and cerebrovascular disease. However, controversies remain as to whether these changes are consequences of the associated obesity or OSA itself results in endocrine and metabolic changes, including impairment of insulin sensitivity, growth hormone, secretion inflammatory cytokines alterations, activation of peripheral sympathetic activity, and hipothalamic-pituitary-adrenal (HPA) axis, that may predispose to vascular disease. Furthermore many cardiovascular risk factors, such as hypertension, obesity, insulin resistance and type 2 diabetes, are strongly associated with OSA. In this article, we will review the evidence and discuss possible mechanisms underlying these links and the pathophysiology of OSA morbidities. (Arq Bras Endocrinol Metab 2007;51/7:1035-1040)

Keywords: Sleep apnea; Insulin resistance; Cardiovascular disease

\section{revisão}

\author{
Gláucia Carneiro \\ FERNANDO FleXA RIBEIRO FILHO \\ SÔNIa MARIA TOGEIRO \\ SÉRGIO TUFIK \\ MARIA TERESA ZANELLA
}

\section{Departamento de Clínica \\ Médica, Disciplina de \\ Endocrinologia (GC, FFRF \& MTZ), \\ e Departamento de \\ Psicobiologia, Disciplina de \\ Biologia e Medicina do Sono \\ (SMT \& ST), Universidade Federal \\ de São Paulo/ Escola Paulista de \\ Medicina, São Paulo, SP.}

Recebido em 15/01/07

Aceito em 10/05/07 
ção atualmente são as co-morbidades associadas, entre elas o maior risco de acidentes de trânsito devido à sonolência excessiva e o risco de doenças cardiovasculares. Vários fatores estruturais têm sido apontados como a etiologia da apnéia obstrutiva do sono, entre eles deposição de gordura na região cervical, hipoplasia de maxila ou mandíbula, macroglossia e hipertrofia de amígdalas. Atualmente, porém, sabe-se que a SAOS não é apenas uma doença local provocada por anormalidades anatômicas e, sim, apresenta características sistêmicas como hipertensão, obesidade central, diabetes e dislipidemia, que sugerem ser uma manifestação da síndrome metabólica. O tratamento de escolha é o uso de máscara (CPAP: continuous positive airway pressure) conectada a um compressor de ar, que provoca pressão positiva e mantém permeável a via aérea superior durante a noite, impedindo seu colapso, principalmente na fase inspiratória.

\section{FISIOPATOLOGIA DA SAOS}

Sabe-se que episódios recorrentes de oclusão das vias aéreas superiores durante o sono provocam alterações, como hipóxia intermitente, severa fragmentação do sono, hipertensão aguda, ativação do sistema nervoso simpático, da atividade inflamatória e do sistema hipotálamo-hipófise-adrenal (HHA), que agravam a obesidade visceral e provocam anormalidades metabólicas como resistência à insulina, diabetes melito (DM), hipertensão arterial sistêmica (HAS) e, conseqüentemente, predispõem a danos vasculares (figura 1). Como a SAOS apresenta forte associação com obesidade, o grande desafio atualmente é realizar estudos que demonstrem que as alterações metabólicas encontradas nos pacientes com apnéia do sono independem da quantidade de gordura corporal.

\section{OBESIDADE E SAOS}

Obesidade, sexo masculino e idade avançada são fatores de risco para síndrome da apnéia obstrutiva do sono $(1,2)$. Aproximadamente $70 \%$ dos pacientes com SAOS são obesos e $40 \%$ dos homens e mulheres obesos apresentam SAOS (3-5). A circunferência da cintura correlaciona-se com a SAOS mais freqüentemente do que o índice de massa corporal, quantidade total de gordura e circunferência do pescoço. Até mesmo em indivíduos não obesos, o aumento da circunferência da cintura prediz SAOS. A cada aumento de $13-15 \mathrm{~cm}$ da circunferência da cintura, eleva-se o risco de SAOS em

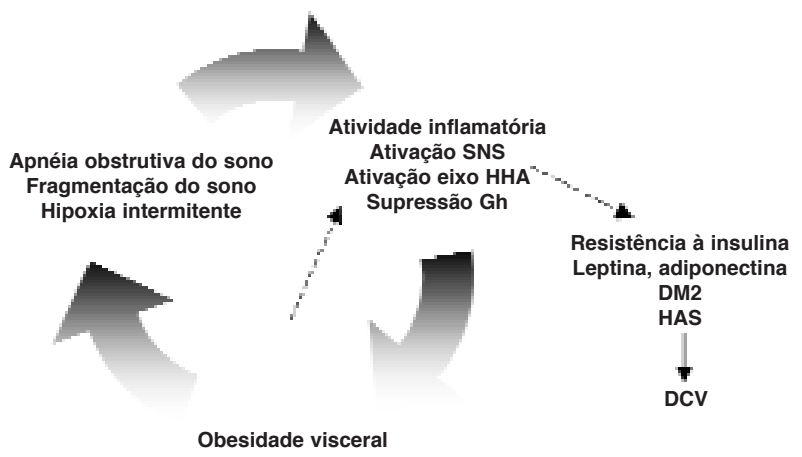

Figura 1. Possíveis interações entre SAOS, resistência à insulina e DCV - Influência da obesidade visceral.

aproximadamente 4 vezes $(6,7)$. Além do mais, o tratamento da SAOS com terapia de pressão contínua positiva das vias aéreas (CPAP) mostrou diminuição do acúmulo da gordura visceral, mesmo na ausência de redução do peso (8). Esses estudos evidenciam que a SAOS está fortemente ligada à obesidade central, e não à gordura corporal total.

A leptina é um hormônio derivado do adipócito que regula o peso corporal através do controle do apetite e gasto energético. Nos pacientes com SAOS, os níveis de leptina estão elevados e diminuem após tratamento com CPAP, mesmo na ausência de redução do peso $(9,10)$. Esses achados sugerem que outros mecanismos, que não a gordura corporal, estariam contribuindo para aumentar os níveis de leptina nos indivíduos com SAOS. A apnéia do sono por si não justifica a elevação dos níveis de leptina, já que estudos anteriores não encontraram correlação entre IAH e leptina. Em contrapartida, sabe-se que a secreção de leptina é estimulada pela ativação do eixo hipotálamohipófise-adrenal na hipóxia e estresse $(11,12)$, e a normalização da ativação do eixo após tratamento com CPAP pode contribuir para reduzir os níveis de leptina nos pacientes tratados. A resistência à leptina também pode favorecer o ganho de peso e ser um marcador para doença cardiovascular, promovendo agregação plaquetária e hipertensão (13).

Outros hormônios secretados pelo tecido adiposo podem provocar alterações metabólicas nos pacientes com SAOS. A adiponectina apresenta correlação negativa com obesidade e resistência à insulina, porém o papel da adiponectina na SAOS ainda permanece controverso. Alguns autores encontraram aumento desse hormônio, enquanto outros relataram níveis de adiponectina diminuídos nos pacientes com o distúrbio respiratório $(14,15)$. 


\section{RESISTÊNCIA À INSULINA (RI) E SAOS}

Uma revisão sistemática, realizada em 2003, mostrou que os estudos observacionais realizados eram inconsistentes para concluir que a apnéia do sono apresentava um papel na patogênese do metabolismo da glicose alterado (16). Stoohs e cols., em 1996, foram os primeiros autores a mostrar que a associação encontrada entre resistência à insulina e apnéia do sono é dependente da massa corporal (17). Entretanto, estudos epidemiológicos recentes afirmaram que a SAOS contribui para a resistência à insulina independentemente do grau e da distribuição da obesidade, idade, sexo, tabagismo e duração do sono (18).

Vgontzas e cols. realizaram polissonografia, dosagens séricas de leptina, citoquinas (IL-6 e TNF- $\alpha$ ), glicemia de jejum, insulina e tomografia computadorizada para avaliação da distribuição da gordura em 14 pacientes com AOS, 11 obesos normais e 12 homens com índice de massa corporal (IMC) normal, e mostraram que os pacientes com SAOS apresentam obesidade centrípeta, maior RI e níveis mais elevados de IL-6, TNF- $\alpha$ e leptina independentemente da obesidade (9). O mesmo grupo de pesquisa estudou o distúrbio do sono em mulheres com síndrome dos ovários policísticos e comparou com mulheres pré-menopausadas controle (19). Os resultados concluíram que a resistência à insulina é um fator de risco maior para apnéia do sono do que o IMC ou testosterona.

Ip e cols. estudaram a associação entre apnéia do sono e resistência à insulina em 270 pacientes consecutivos sem diabetes, obesos ou não, que realizaram polissonografia. Pacientes com apnéia do sono $(\mathrm{n}=$ 185; IAH > 5) apresentaram níveis mais elevados de glicemia de jejum e HOMA-IR ( $<<0,001)$ comparados com os pacientes sem apnéia do sono $(\mathrm{n}=$ $85)$. Os pacientes com apnéia do sono eram mais velhos e obesos, porém a regressão linear múltipla mostrou que o IAH e a saturação mínima de oxigênio contribuíram significativamente com a insulina de jejum e HOMA-IR, independentemente do IMC e da idade dos pacientes (20).

Tassone e cols. mostraram que os pacientes com SAOS $(\mathrm{n}=30)$ apresentam menor sensibilidade à insulina, calculada pelo ISI, do que o grupo controle sem AOS $(\mathrm{n}=27)$, mesmo após ajuste para idade, IMC e relação cintura-quadril (21).

Além da resistência à insulina, alguns autores evidenciaram maior prevalência de diabetes melito tipo 2 em pacientes com SAOS e redução dos níveis de HbAlc após tratamento com CPAP (22). Babu e cols. estudaram 25 pacientes com DM2 descompensado
(HbAlc > 7) e apnéia do sono, e observaram que houve controle do DM2 após 3 meses de tratamento com CPAP (23).

Estudos sobre o efeito do tratamento da SAOS com CPAP na resistência à insulina são conflitantes (16). Justificativas para os resultados negativos são a má aderência dos pacientes ao CPAP, duração curta dos estudos, ou devido à AOS ser uma doença crônica e poder ocasionar, com o tempo, alterações metabólicas como a redistribuição da gordura central, e este ser um componente para o tratamento da SAOS aparentemente inefetivo em modificar a sensibilidade à insulina.

Várias hipóteses podem ser realizadas para explicar os mecanismos da piora da resistência à insulina nos indivíduos obesos com apnéia do sono predispondo às alterações vasculares. A hipóxia intermitente, o déficit de sono, a hipercitoquinemia (TNF- $\alpha$ e IL6), a disfunção do eixo somatotrófico e corticotrófico e a ativação do sistema nervoso simpático estão diretamente envolvidos na diminuição da sensibilidade à insulina, e essas alterações são encontradas em indivíduos obesos e com SAOS.

\section{Hipóxia e o déficit de sono}

Apesar de os estudos evidenciarem uma associação independente entre apnéia do sono e disfunção metabólica, não há estudos prospectivos para saber se essa associação é causal ou se ocorre via fragmentação do sono e hipóxia intermitente. Os efeitos metabólicos da hipóxia intermitente já foram estudados em humanos e animais $(24,25)$. A hipóxia poderia levar a uma diminuição da secreção e ação da insulina, através da diminuição da produção de ATP pela célula B e redução da atividade tirosinoquinase dos receptores de insulina. Além dos efeitos da hipóxia intermitente, há evidências de que anormalidades no sono, por si, podem alterar o metabolismo da glicose (26). A restrição do sono $(4 \mathrm{~h} /$ dia durante 6 noites $)$ em indivíduos normais piorou a tolerância à glicose, aumentou os níveis de cortisol, ativou o sistema nervoso simpático e provocou uma resposta inflamatória. As evidências acima sugerem que a hipóxia e o déficit de sono podem contribuir independentemente para alterações no metabolismo da glicose nos pacientes com apnéia do sono.

\section{Marcadores inflamatórios}

A obesidade pode induzir o aumento de citoquinas e piorar as alterações metabólicas encontradas nos pacientes com apnéia. Entretanto, vários autores já demonstraram que níveis de citoquinas estão elevados 
em pacientes com SAOS independentemente da gordura corporal. Portanto, é aceitável que o aumento dos marcadores inflamatórios na obesidade não está relacionado apenas com a adiposidade, mas pode também estar associado à SAOS.

Sabe-se que a hipoxemia da alta atitude e a privação do sono provocam aumento de IL6 e PCR em indivíduos normais (27). Portanto, nos pacientes com SAOS, a hipoxemia que ocorre repetidamente durante o sono, acompanhada da privação do sono, pode induzir a um estado sistêmico inflamatório, evidenciado pela elevação de PCR, amiloide A, IL-6 e TNF- $\alpha(9,28)$.

$\mathrm{O}$ TNF- $\alpha$ elevado contribui para a resistência à insulina provocando down-regulation no GLUT4 e inibindo a atividade e sinalização do receptor da insulina. A IL-6 secretada pelo tecido adiposo induz à secreção hepática de PCR, e ambos os marcadores inflamatórios estão associados com a obesidade, AOS e doenças cardiovasculares (29).

Além de aumentar a resistência à insulina, provocar dislipidemia e disfunção endotelial, há evidências de que as citoquinas inflamatórias (IL-1B, IL-6, TNF- $\alpha$ ) também estão envolvidas na regulação do sono, e a secreção endógena ou administração exógena está associada com sonolência e fadiga (9).

Etanercept é uma medicação antiinflamatória que bloqueia a ligação do TNF- $\alpha$ ao receptor e é utilizada para artrite reumatóide na dose de $25 \mathrm{mg} 2$ vezes por semana por via subcutânea. Foi realizado um estudo em 8 pacientes obesos com apnéia do sono que receberam etanercept e/ou placebo. Foi observada redução da sonolência, do IAH e dos níveis de IL-6, mas não houve alteração nos níveis de glicemia, insulina e adiponectina nos pacientes que receberam o medicamento (30).

Esses dados corroboram a hipótese de que a inflamação é um importante mecanismo na patogênese da apnéia do sono e sonolência, resistência à insulina e obesidade visceral, e todos esses mecanismos promovem aterosclerose, doenças cardiovasculares e morte prematura.

\section{Sistema nervoso simpático}

Vários estudos evidenciaram a ativação do SNS na SAOS $(31,32)$. Concentrações de catecolaminas sérica e na urina de $24 \mathrm{~h}$ e a atividade nervosa simpática muscular estão elevadas em pacientes com SAOS, e essas respostas são revertidas após tratamento com CPAP por mais de $4 \mathrm{~h}(33)$.

O sistema nervoso simpático pode influenciar na gênese da hipertensão arterial provocando vaso- constrição, e na homeostase da glicose aumentando a glicogenólise, a neoglicogênese e a secreção de glucagon. Em 2003, Chasens e cols. propuseram que a AOS provoca diabetes melito tipo 2 via ativação do sistema nervoso simpático (34).

\section{Eixo HHA e somatotrófico}

A ativação do eixo corticotrófico pode ser um dos fatores que contribuem para o desenvolvimento da resistência à insulina nos pacientes com AOS.

A hipóxia intermitente, fragmentação e privação do sono provocam liberação de cortisol pulsátil e ativação autonômica (35). Entretanto, a literatura varia em relação aos efeitos da AOS no eixo HHA. Alguns autores demonstraram que o eixo hipotálamohipófise adrenal está ativado $(31,36,37)$, mas outros não (38). Além disso, ainda é necessário esclarecer os efeitos do CPAP no eixo HHA $(38,39)$. A maioria desses estudos é limitada, pois apenas uma única medida do cortisol foi realizada e, conseqüentemente, não detectaram alterações clínicas do ritmo do cortisol.

A secreção do hormônio de crescimento $(\mathrm{GH})$ ocorre principalmente à noite, durante os estágios III e IV do sono de ondas lentas no eletroencefalograma. Nos pacientes com apnéia do sono, ocorre diminuição ou abolição das ondas lentas e, conseqüentemente, diminuição da secreção do GH. Esses dados confirmam os achados de que a secreção intermitente do GH está abolida nos pacientes com AOS e é restaurada após tratamento que elimina a obstrução das vias aéreas $(38,40)$. Indivíduos adultos com deficiência de $\mathrm{GH}$ apresentam resistência à insulina e características da síndrome metabólica.

\section{DCV e SAOS}

A doença cardiovascular resultante da SAOS inclui hipertensão arterial sistêmica, insuficiência cardíaca esquerda, infarto do miocárdio, arritmias e hipertensão pulmonar, podendo culminar com morte súbita. Mais de $50 \%$ dos pacientes com apnéia são hipertensos. Por outro lado, cerca de $40 \%$ dos pacientes hipertensos podem ter apnéia do sono não diagnosticada. Freqüentemente esses pacientes apresentam hipertensão de difícil controle e observa-se redução dos níveis pressóricos após tratamento com CPAP (41). Lavie e cols. (42) mostraram que a prevalência de hipertensão nos pacientes com SAOS é de 60\% e a cada episódio de apnéia por hora (IAH) durante o sono, aumenta em $1 \%$ o risco de hipertensão arterial. As possíveis explicações para a elevação da pressão arterial nesses pacientes são: 
- Agudamente, durante a apnéia ocorre hipoxemia, hipercapnia e estímulo do sistema nervoso simpático com conseqüente vasoconstrição periférica. Há, também, diminuição da pressão negativa intratorácica e conseqüente diminuição da pré e pós-carga cardíaca. Após a restauração da respiração, aumenta-se o volume circulante (pré-carga) na vigência da vasoconstrição, provocando elevações repetitivas da PA durante a noite.

- Cronicamente, podem ser observadas elevações sustentadas da PA por estímulo da atividade simpática, redução da atividade parassimpática, diminuição do óxido nítrico e liberação de endotelina. Recentemente, foi sugerido que a ativação do ACTH devido ao estresse estimula a síntese e secreção de aldosterona e cortisol (43). O hiperaldosteronismo pode ser uma causa de hipertensão resistente nos pacientes com AOS. A ativação do sistema renina-angiotensina, inflamação, resistência à insulina, diminuição na sensibilidade dos baroceptores, disfunção endotelial, estresse oxidativo e hiperleptinemia também podem estar implicados no desenvolvimento da hipertensão arterial (44).

Os mecanismos propostos para explicar o maior risco cardiovascular nos indivíduos com AOS ainda são controversos devido à influência da obesidade, mas atualmente há evidências consistentes de que os pacientes com SAOS não tratados apresentam morbidades para doenças cardiovasculares, independentemente da obesidade. Uma recente publicação com seguimento dos pacientes com SAOS durante 10 anos mostrou que houve uma relação entre severidade da apnéia e risco cardiovascular fatal e não fatal, independentemente do peso e da idade, e o tratamento com CPAP reduziu significativamente os eventos cardiovasculares $(45)$.

\section{CONCLUSÃo}

Muitas evidências sugerem que a apnéia do sono é uma doença metabólica e sistêmica e está associada à morbidade e mortalidade, principalmente por acidente de trânsito e doença cardiovascular. A fisiopatologia da apnéia do sono permanece obscura e a maioria dos tratamentos, atualmente, são mecânicos com respostas variadas e baixa aderência. Além disso, os custos das complicações são elevados. Por esses motivos, uma avaliação rigorosa deve ser realizada nos pacientes de risco e uma melhor compreensão dessa patologia pode propiciar métodos mais efetivos para tratamento e prevenção dessa co-morbidade e suas complicações.

\section{REFERÊNCIAS}

1. Block AJ, Boysen PG, Wynne JW, Hunt LW. Sleep apnea, hipopnea and oxygen desaturation in normal subjects: a strong male predominance. N Engl J Med 1979;300:513-7.

2. Davies RJ, Stradling JR. The relationship between neck circumference, radiographic pharyngeal anatomy, and the obstructive sleep apnea syndrome. Eur Resp J 1990;3:509-14.

3. Vgontzas AN, Tan TL, Bixler EO, Martin LF, Shubert D, Kales A. Sleep apnea and sleep disruption in obese patients. Arch Intern Med 1994;154(15):1705-11.

4. Richman RM, Elliott LM, Burns CM, Bearpark HM, Steinbeck KS, Caterson ID. The prevalence of obstructive sleep apnoea in an obese female population. Int J Obes Relat Metab Disord 1994;18(3):173-7.

5. Resta O, Foschino-Barbaro MP, Legari G, Talamo S, Bonfitto $P$, Palumbo $A$, et al. Sleep-related breathing disorders, loud snoring and excessive daytime sleepiness in obese subjects. Int J Obes Relat Metab Disord 2001;25(5):669-75.

6. Grunstein R, Wilcox I, Yang TS, Gould Y, Hedner J. Snoring and sleep apnoea in men: association with central obesity and hypertension. Int $J$ Obes Relat Metab Disord 1993:17(9):533-40.

7. Young T, Palta M, Dempsey J, Skatrud J, Weber S, Badr S. The occurrence of sleep-disordered breathing among middleaged adults. N Engl J Med 1993;328(17):1230-5.

8. Chin K, Shimizu K, Nakamura T, Narai N, Masuzaki H, Ogawa $Y$, et al. Changes in intra abdominal visceral fat and serum leptin levels in patients with obstructive sleep apnea syndrome following nasal continuous positive airway pressure therapy. Circulation 1999;100:706-12.

9. Vgontzas AN, Papanicolaou DA, Bixler EO, Hopper K, Lotsikas $A$, Lin HM, et al. Sleep apnea and daytime sleepiness and fatigue: relation to visceral obesity, insulin resistance, and hypercytokinemia. J Clin Endocrinol Metab 2000;85(3):1151-8.

10. Ip MSM, Lam KSL, Ho CM, Tsang KWT, Lam WK. Serum leptin and vascular risk factors in obstructive Sleep Apnea. Chest 2000;118:580-6.

11. Tshop M, Strasburger CJ, Topfer M, Hautmann H, Riepl R, Fisher R, et al. Influence of hypobaric hypoxia on leptin levels in men. Int $\mathbf{J}$ Obes Relat Metab Disord 2000;24:S151.

12. Sandoval DA, Davis SN. Leptin: metabolic control and regulation. J Diabetes Complications 2003;17:108-13.

13. Konstantinides S, Schafer K, Koschnick S, Loskutoff DJ. Leptin dependent platelet aggregation and arterial thrombosis suggests a mechanism for atherothrombotic disease in obesity. J Clin Invest 2001;108:1533-40.

14. Zhang $X L$, Yin KS, Mao $H$, Wang $H$, Yang $Y$. Serum adiponectin level in patients with obstructive sleep apnea hypopnea syndrome. Chin Med J (Engl) 2004:117:1603-6.

15. Wolk R, Svatikova A, Nelson CA, Gami AS, Govender K, Winnicki $M$, et al. Plasma levels of adiponectin, a novel adipocytederived hormone, in sleep apnea. Obes Res 2005;13:186-90.

16. Punjab NM, Ahmed MM, Polotsky VY, Beamer BA, O'Donnel $\mathrm{CP}$. Sleep-disordered breathing glucose intolerance, and insulin resistance. Respir Physiol Neurobiol 2003;136:167-78.

17. Stoohs RA, Facchini F, Guilleminault C. Insulin resistance and sleep-disordered breathing in health humans. Am J Respire Crit Care Med 1996;154:170-4.

18. Punjab NM, Shahar E, Redline S, Gttieb DJ, Givelber R, Resnick $\mathrm{HE}$; for the Sleep Heart Study Investigator. Sleep-disordered breathing, glucose intolerance and insulin resistance. The Sleep Heart Health Study. Am J Epidemiol 2004;160:521-30.

19. Vgontzas AN, Legro VS, Bixler EO, Grayev A, Kales A, Chrousos GP. Polycystic ovary syndrome is associated with obstructive sleep apnea and daytime sleepiness: role of insulin resistance. J Clin Endocrinol Metab 2001;86:517-20.

20. Ip MSM, Lam B, Ng MMT, Lam WKT, Tsang KWT, Lam KSL. Obstructive Sleep apnea is independently associated with insulin resistance. Am $J$ Respir Crit Care Med 2002;165:670-6. 
21. Tassone F, Lanfranco F, Gianotti L, Pivetti S, Navone F, Rosseto $R$, et al. Obstructive sleep apnoea syndrome impairs insulin sensitivity independently of anthropometric variables. Clin Endocrinol 2003;59:374-9.

22. Brooks B, Cistulli PA, Borkman M, Ross G, McGhee S, Grunstein RR, et al. Obstructive sleep apnea in obese non insulin dependent diabetic patient: effect of continuous positive airway pressure treatment o insulin responsiveness. J Clin Endocrinol Metab 1994:79:1681-5.

23. Babu AR, Herdeger J, Fogefeld L, Shott S, Mazzone T. Type 2 diabetes, glycemic control, and continuous positive airway pressure in obstructive sleep apnea. Arch Intern Med 2005;165(4):447-52.

24. Larsen JJ, Hansen JM, Olsen NV, Galbo H, Dela F. The effect of altitude hypoxia on glucose homeostasis in man. J Physiol 1997:504:241-9.

25. Polotsky VY, Li J, Punjab N, Rubin A, Smith PL, Schwartz AR, et al. Intermittent hypoxia increases insulin resistance in genetically obese mice. J Physiol 2003;552:253-64

26. Spiegel K, Leproult R, Van Cauter E. Impact of sleep debt on metabolic and endocrine function. Lancet 1999;354:1435-9.

27. Hartmann G, Tschop M, Fischer R, Bidlingmaier C, Riepl R, Tschop K, et al. High altitude increases circulating interleukin6 , interleukin-1 receptor antagonist and C- reative protein. Cytokine 2000;12:246-52.

28. Shamsuzzaman AS, Winnicki M, Lanfranchi $P$, Wolk R, Kara T, Accurso V, et al. Elevated C- reactive protein in patients with obstructive sleep apnea. Circulation 2002;105:2462-4.

29. Hotamisligil GS. Mechanism of TNF-alpha induced insulin resistance. Exp Clin Endocrinol Diabetes 1999;107:11952.

30. Vgontzas AN, Zoumakis E, Lin HM, Bixler EO, Trakada G, Chrousos GP. Marked decrease in sleepiness in patients with Sleep apnea by etanercept, a tumor necrosis Factor-alpha antagonist. J Clin Endocrinol Metab 2004;89(9):4409-13.

31. Bratel T, Wennlund A, Carlstrom K. Pituitary reactivity, androgens and catecholamines in obstructive sleep apnoea. Effects of continuous positive airway pressure treatment (CPAP). Respiratory Medicine 1999;93:1-7.

32. Zwillich CW. Sleep apnoea and autonomic function. Thorax 1998;53:20-4

33. Waradekar NV, Sinoway LI, Zwillich CW, Leuenberger UA. Influence of treatment on muscle sympathetic nerve activity in sleep apnea. Am J Respir Crit Care Med 1996;153(4 Pt 1):1333-8.

34. Chasens ER, Weaver TE, Umlauf MG. Insulin resistance and obstructive sleep apnea: is increased sympathetic stimulation the link? Biol Res Nurs 2003;5:87-96
35. Spath-Schwalbe E, Gofferje M, Kern W, Born J, Fehm HL Sleep disruption alters nocturnal ACTH and cortisol secretory patterns. Biol Psychiatry 1991;29:575-84.

36. Gianotti L, Pivetti S, Lafranco F, Tassone F, Navone F, Vittori $E$, et al. Concomitant impairment of growth hormone secretion and peripheral sensitivity in obese patient with obstructive sleep apnea syndrome. J Clin Endocrinol Metab 2002;87:5052-7.

37. Lanfranco F, Gianotti L, Pivetti S, Navone F Rosseto R, Tassone $\mathrm{F}$, et al. Obese patients with obstructive sleep apnoea syndrome show a peculiar alteration of the corticotroph but not of the thyrotroph and -lactotroph function. Clin Endocrinol (Oxf) 2004:60(1):41-8.

38. Grunstein RR, Handelsman DJ, Lawrence SJ, Blackwell C, Caterson J, Sullivan CE. Neuroendocrine dysfunction in sleep apnea: reversal by continuous positive airway pressure therapy. J Clin Endocrinol Metab 1989;68:352-8.

39. Cahan C, Arafah B, Decker MJ, Arnold JL, Strohl KP. Adrenal steroids in sleep apnea before and after nCPAP treatment. Am Rev Respir Dis 1991;143:A382.

40. Krieger J. Sleep apnea syndrome in adults. Bull Eur Physiopathol Respir 1986;22:147.

41. Wilcox I, Grunstein RR, Hedner JA, Doyle J, Collins FL, Fletcher PJ, et al. Effect of nasal continuous positive airway pressure during sleep on 24-hour blood pressure in obstructive sleep apnea. Sleep 1993;16:539-44.

42. Lavie $P$, Herer $P$, Hoffstein V. Obstructive sleep apnoea syndrome as a risk factor for hypertension: population study. Brit Med J 2000;320:479-82.

43. Goodfriend TL, Calhoun DA. Resistant hypertension, obesity, sleep apnea, and aldosterone: theory and therapy. Hypertension 2004;43:518-24.

44. Peppard PE, Young T, Palta M, Skatrud J. Prospective study of the association between sleep-disordered breathing and hypertension. N Engl J Med 2000;342:1378-84.

45. Marin JM, Carrizo SJ, Vicente E, Agusti AGN Long-term cardiovascular outcomes in men with obstructive sleep apnoea hypopnoea with or without treatment with continuous positive airway pressure: an observational study. Lancet 2005;365:1046-53.

Endereço para correspondência:

Gláucia Carneiro

Rua Correia de Lemos 327, apto 132

04140-000 São Paulo, SP 